\title{
Selective photoinactivation of Candida albicans in the non-vertebrate host infection model Galleria mellonella
}

José Chibebe Junior ${ }^{1,2,3 \dagger}$, Caetano P Sabino ${ }^{4,5+}$, Xiaojiang Tan ${ }^{6,2 \dagger}$, Juliana C Junqueira ${ }^{{ }^{*}}$, Yan Wang ${ }^{2,7}$, Beth B Fuchs ${ }^{2}$, Antonio OC Jorge ${ }^{1}$, George P Tegos ${ }^{4,8,9}$, Michael R Hamblin ${ }^{4,9,10}$ and Eleftherios Mylonakis ${ }^{2,11}$

\begin{abstract}
Background: Candida spp. are recognized as a primary agent of severe fungal infection in immunocompromised patients, and are the fourth most common cause of bloodstream infections. Our study explores treatment with photodynamic therapy (PDT) as an innovative antimicrobial technology that employs a nontoxic dye, termed a photosensitizer (PS), followed by irradiation with harmless visible light. After photoactivation, the PS produces either singlet oxygen or other reactive oxygen species (ROS) that primarily react with the pathogen cell wall, promoting permeabilization of the membrane and cell death. The emergence of antifungal-resistant Candida strains has motivated the study of antimicrobial PDT (aPDT) as an alternative treatment of these infections. We employed the invertebrate wax moth Galleria mellonella as an in vivo model to study the effects of aPDT against C. albicans infection. The effects of aPDT combined with conventional antifungal drugs were also evaluated in G. mellonella.

Results: We verified that methylene blue-mediated aPDT prolonged the survival of C. albicans infected G. mellonella larvae. The fungal burden of $G$. mellonella hemolymph was reduced after aPDT in infected larvae. A fluconazoleresistant $C$. albicans strain was used to test the combination of aPDT and fluconazole. Administration of fluconazole either before or after exposing the larvae to aPDT significantly prolonged the survival of the larvae compared to either treatment alone.
\end{abstract}

Conclusions: G. mellonella is a useful in vivo model to evaluate aPDT as a treatment regimen for Candida infections. The data suggests that combined aPDT and antifungal therapy could be an alternative approach to antifungalresistant Candida strains.

Keywords: Candida albicans, Photodynamic therapy, Galleria mellonella

\section{Background}

Candida albicans and other Candida species commonly colonize the epithelial surfaces of the human body [1]. One-half of humans have oral cavities colonized by Candida species in a commensal relationship with the host [2]. Although few healthy carriers develop clinical candidiasis, when the host becomes immunocompromised due to cancer, HIV/AIDS, diabetes, major surgery, transplantation of solid organs or hematopoietic stem cells, these opportunistic pathogens can cause superficial

\footnotetext{
* Correspondence: julianacjunqueira@hotmail.com

${ }^{\dagger}$ Equal contributors

'Department of Biosciences and Oral Diagnosis, Univ Estadual Paulista/ UNESP, São José dos Campos, SP 12245000, Brazil

Full list of author information is available at the end of the article
}

infections that may be cutaneous, subcutaneous or mucosal. In progressive cases, the fungus can penetrate the epithelial surface and be disseminated by the bloodstream with serious consequences [1,3-7].

C. albicans is the most common species isolated from superficial and systemic candidiasis and it is considered the most pathogenic species of the Candida genus [5,8-11]. In vitro investigations indicate that C. albicans expresses higher levels of putative virulence factors compared to other Candida species. It has been proposed that several virulence factors are involved in the pathogenicity of C. albicans, such as adhesion to host surfaces, hyphal formation and secretion of proteinases [11]. In addition, C. albicans cells employ mechanisms

\section{Biomed Central}

(c) 2013 Chibebe Junior et al.; licensee BioMed Central Ltd. This is an Open Access article distributed under the terms of the Creative Commons Attribution License (http://creativecommons.org/licenses/by/2.0), which permits unrestricted use, distribution, and reproduction in any medium, provided the original work is properly cited. 
that protect of the fungal cells from the host immune system, including an efficient oxidative stress response $[12,13]$. When immunocompetent individuals are infected by fungi, macrophages and neutrophils generate reactive oxygen species (ROS), such as superoxide radicals and hydrogen peroxide that damage cellular components of C. albicans, inclusive of proteins, lipids and DNA. The production of ROS is an important mechanism of host defense against fungal pathogens [13], damaging cells enough to cause cell death of phagocytosed fungal cells $[12,14]$.

Treatment of fungal infections, especially invasive ones, is considered difficult due to the limited availability of antifungal drugs and by the emergence of drugresistant strains. The development of new antifungal agents and new therapeutic approaches for fungal infections are therefore urgently needed $[4,8,15]$. Photodynamic therapy (PDT) is an innovative antimicrobial approach that combines a non-toxic dye or photosensitizer (PS) with harmless visible light of the correct wavelength. The activation of the PS by light results in the production of ROS, such as singlet oxygen and hydroxyl radicals, that are toxic to cells $[6,16]$. PDT is a highly selective modality because the PS uptake occurs mainly in hyperproliferative cells and cell death is spatially limited to regions where light of the appropriate wavelength is applied. As microbial cells possess very fast growth rates, much like that of malignant cells, PDT has been widely used for microbial cell destruction [17]. Several in vitro studies have shown that PDT can be highly effective in the inactivation of $C$. albicans and other Candida species. Therefore, antifungal PDT is a subject of increasing interest especially against Candida strains resistant to conventional antifungal agents [16].

Galleria mellonella (the greater wax moth) has been successfully used to study pathogenesis and infection by different fungal species, such as Candida albicans, Cryptococcus neoformans, Fusarium oxysporum, Aspergillus flavus and Aspergillus fumigatus [18]. Recently, our laboratory was the first to describe G. mellonella as an alternative invertebrate model host to study antimicrobial PDT alone or followed by conventional therapeutic antimicrobial treatments [19]. We demonstrated that after infection by Enterococcus faecium, the use of antimicrobial PDT prolonged larval survival. We have also found that aPDT followed by administration of a conventional antibiotic (vancomycin) was significantly effective in prolonging larval survival even when infected with a vancomycin-resistant E. faecium strain.

In this study, we go on to report the use of the invertebrate model G. mellonella as a whole animal host for the in vivo study of antifungal PDT, as well as the study of combined therapy using PDT and a conventional antifungal drug.

\section{Methods}

Microbial strains and culture conditions

The C. albicans strains used in this study were Can14 and Can37. C. albicans Can14 is a wild-type strain SC5314 [20] and C. albicans Can37 is a fluconazole resistant clinical isolate from a patient with oropharyngeal candidiasis [3]. C. albicans Can37 was identified by growth on Hicrome Candida (Himedia, Munbai, India), germ tube test, clamydospore formation on corn meal agar, and API20C for sugar assimilation (BioMerieux, Marcy Etoile, France). Susceptibility pattern to fluconazole was determined by the broth microdilution assay according to the Clinical and Laboratory Standards Institute (CLSI).

Strains were stored as frozen stocks with $30 \%$ glycerol at $-80^{\circ} \mathrm{C}$ and subcultured on YPD agar plates (1\% yeast extract, $2 \%$ peptone, and $2 \%$ dextrose) at $30^{\circ} \mathrm{C}$. Strains were routinely grown in YPD liquid medium at $30^{\circ} \mathrm{C}$ in a shaking incubator.

\section{Fungal inocula preparation}

C. albicans cells were grown in YPD at $30^{\circ} \mathrm{C}$ overnight. Cells were collected with centrifugation and washed three times with PBS. Yeast cells were counted using a hemocytometer. The cell number was confirmed by determining colony-forming units per $\mathrm{mL}(\mathrm{CFU} / \mathrm{mL})$ on YPD plates.

\section{Inoculation of G. mellonella with C. albicans strains}

G. mellonella (Vanderhorst Wholesale, St. Marys, OH, USA) in the final larval stage were stored in the dark and used within 7 days from shipment. Sixteen randomly chosen G. mellonella larvae with similar weight and size (250-350 mg) were used per group in all assays. Two control groups were included: one group was inoculated with PBS to observe the killing due to physical trauma, and the other received no injection as a control for general viability.

A Hamilton syringe was used to inject $5 \mu \mathrm{L}$ inoculum aliquots into the hemocoel of each larvae via the last left proleg containing $10^{6} \mathrm{CFU} /$ larvae of C. albicans cells suspended in PBS. After injection, larvae were incubated in plastic containers at $37^{\circ} \mathrm{C}$ and monitored for survival daily.

\section{Chemicals and photosensitizer}

Methylene blue (MB, Sigma, St Louis, MO) was used at a final working concentration of $1 \mathrm{mM}$. The dye was dissolved in distilled and deionized filter sterilized water $\left(\mathrm{ddH}_{2} \mathrm{O}\right)$. For each experiment, a new PS solution was prepared daily. Fluconazole (Sigma-Aldrich, Steinheim, Germany) was dissolved in $\mathrm{ddH}_{2} \mathrm{O}$ and injected in $G$. mellonella at a concentration of $14 \mathrm{mg} / \mathrm{Kg}$. 


\section{Antimicrobial photodynamic therapy}

The G. mellonella larvae were injected with $10 \mu \mathrm{L}$ of a $1 \mathrm{mM}$ solution of MB 90 min after the Candida infection and the PS was allowed to disperse for 30 min into the insect body in the dark, prior to the light irradiation.

A broad-band non coherent light source (LumaCare, Newport Beach, CA) was used for light delivery. This device was fitted with a $660 \pm 15 \mathrm{~nm}$ band-pass filter probe that was employed to produce a uniform spot for illumination. The optical power was measured using a power meter (PM100D power/energy meter, Thorlabs, Inc., Newton, NJ).

\section{Antifungal administration}

For the study of aPDT combined with conventional antifungal drug, fluconazole $(14 \mathrm{mg} / \mathrm{kg})$ was injected immediately before or after the exposure of larvae to light. As a control, a group of the larvae received an injection containing PBS, in lieu of fluconazole.

\section{G. mellonella survival assays}

After aPDT or combined treatment of aPDT with fluconazole, larvae were observed every $24 \mathrm{~h}$, and considered dead when they displayed no movement in response to touch. Survival curves were plotted and statistical analysis was performed by the Log-rank (Mantel-Cox) test using Graph Pad Prism statistical software. A $P$ value $<0.05$ was considered statistically significant. All experiments were repeated at least twice, representative experiments are presented.

\section{Persistence of C. albicans in the hemolymph of $G$. mellonella}

The number of fungal cells recovered from the hemolymph of G. mellonella infected by C. albicans Can37 was measured immediately after larvae were exposed to aPDT and to combined treatment (aPDT and fluconazole). Three surviving larvae per group were bled by insertion of a lancet into the hemocoel. Hemolymph from 3 larvae was pooled into $1.5 \mathrm{ml}$ Eppendorf tubes in a final volume of approximately $80 \mu \mathrm{L}$. Then, the hemolymph was serially diluted and plated on Sabouraud dextrose agar supplemented with chloramphenicol $(100 \mathrm{mg} / \mathrm{L})$. Plates were incubated aerobically at $37^{\circ} \mathrm{C}$ for $24 \mathrm{~h}$, and colonies were counted in each pool (CFU/pool). The groups exposed to aPDT were compared to the control groups by Student $t$ test. Difference in the number of CFUs were considered statistically significant at $P<0.05$. The experiments were repeated at least twice and representative experiments are presented. Three polls per group were performed in each experiment.

\section{Results}

We previously described the utility of the G. mellonella model host to assess antibacterial PDT efficacy against E. faecium [19]. In this study we explored the potential of this model using antifungal therapy against one of the most common opportunistic fungal pathogens $C$. albicans. Briefly, after 90 min of Candida infection, G. mellonella larvae were treated with PDT mediated by MB and red light according to the methods described.

As a first step in exploring the optimal dose-response to $\mathrm{MB}$ mediated-PDT, we evaluated 10 groups of larvae that were infected with the wild-type strain of $C$. albicans (Can14) and received MB (1 mM) injection. We gradually increased the light exposure time. More specifically, eight groups were exposed to red light at different fluences $(0.9$, $1.8,3.6,5.4,7.2,10.8,14.4$ and $18 \mathrm{~J} / \mathrm{cm}^{2}$, corresponding to $30,60,120,180,240,360,480$ and $600 \mathrm{~s}$ of irradiation), while two control groups received injection of PBS or MB with no light exposure. After irradiation, the survival rate of G. mellonella was assessed $24 \mathrm{~h}$ post C. albicans infection. The best survival rate was reached with the lowest dose and $30 \mathrm{~s}$ of irradiation time (data not shown).

As a second step, a finer evaluation to establish the optimum light dosimetry was performed. Eight further groups were employed to analyze the photodynamic effects at $15,30,45,60,75,90,105$ and $120 \mathrm{~s}$ of irradiation $(0.45,0.9,1.35,1.8,2.25,2.7$ and $3.6 \mathrm{~J} / \mathrm{cm} 2)$ and once again $0.9 \mathrm{~J} / \mathrm{cm}^{2}$ (30 $\mathrm{s}$ of irradiation) provided the best survival rate (Figure 1).

As a third step, a further comprehensive experimental procedure was designed to assess the effects of aPDT, mediated by the optimum dose $(1 \mathrm{mM} \mathrm{MB}$ and red light at $0.9 \mathrm{~J} / \mathrm{cm}^{2}$ ), on host curve survival when infected by the wild-type strain C. albicans Can14 and the fluconazole resistant isolate $C$. albicans Can37. We observed that MBmediated aPDT, prolonged the larval survival when compared to non-PDT treated larvae, however a statistically significant difference between PDT and control groups was observed only for C. albicans Can14 (Figure 2).

Since it was observed that fluconazole resistant strain (Can37) showed reduced sensitivity to PDT, we evaluated the number of CFU within the hemolymph to determine if the fungal burden was reduced even if survival was not significantly increased. We compared the hemolymph burden of aPDT-treated larvae with non-treated larvae and a significant reduction in the CFU number was observed post-PDT treatment (Figure 3). These results confirmed that aPDT was able to reduced fungal cell viability $(0.2$ Log) immediately upon light exposure, suggesting that singlet oxygen and other ROS were produced, leading to cell damage [21,22].

The reduced fungal burden indicates that the aPDT treated cells are potentially damaged and thus the survival might be altered by the addition of another cell membrane directed bombarding compound, a structure important for the maintenance of cell wall integrity. Hence, we investigated the effects of combined treatment of aPDT with fluconazole, a compound that 


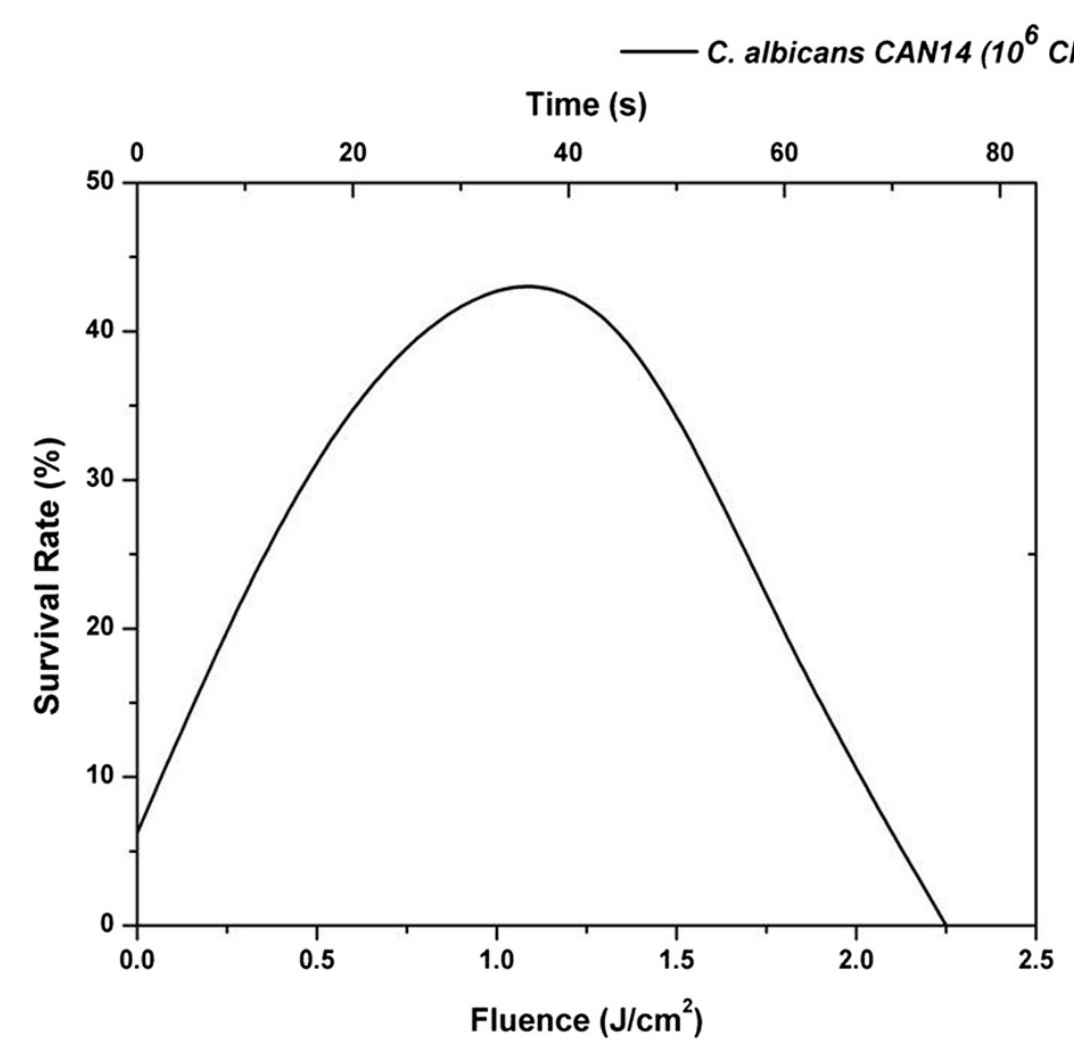

Figure 1 Dose-response $24 \mathrm{~h}$ after aPDT in G. mellonella infected by C. albicans Can 14. Larvae were infected with $1 \times 10^{6}$ CFU/larva of C. albicans Can14. The best survival rate was found when the fluence of $0.9 \mathrm{~J} / \mathrm{cm}^{2}$ was applied.

targets P450 and affects ergesterol synthesis, a major component of the cell membrane. This antifungal agent is used extensively because of its low host toxicity to treat fungal infections. One of the mechanisms that can be used by $C$. albicans to develop resistance to fluconazole is related to the overexpression of cell membrane multidrug efflux systems $[23,24]$. Based on the hypothesis that aPDT could damage the cell membrane of $C$. albicans, producing increased membrane permeability [25] and possibly damaging efflux pumps, we used G. mellonella-C. albicans system to assess the sequential combination of PDT with fluconazole. G. mellonella were inoculated with $1.41 \times 10^{6} \mathrm{CFU} /$ larva to infect the larvae with the fluconazole-resistant $C$. albicans strain (C. albicans Can37). Larvae treated only with PDT or only with fluconazole did not show significantly prolonged larval survival. The sequential combination with fluconazole, before or after PDT, significantly increased larvae survival in both assays (Figure 4). These results suggest that aPDT increases the susceptibility of $C$. albicans Can37 to fluconazole.

\section{Discussion and conclusion}

In this study we used the invertebrate model G. mellonella for the in vivo study of antifungal PDT. We verified that aPDT prolonged the survival of G. mellonella caterpillars infected by $C$. albicans and reduced the fungal burden in the hemolymph of these animals. In addition, we used a fluconazole-resistant $C$. albicans strain to test the combination of aPDT and fluconazole. The data presented here demonstrated that aPDT increased the susceptibility of $C$. albicans to fluconazole.

The increased numbers of fungal infections and the subsequent need for high-cost and time-consuming development of new antimicrobial strategies and anti-infectives has emerged as a major problem among infectious diseases researchers and clinicians $[6,26]$. Antimicrobial PDT is one of the most promising alternative countermeasures for cutaneous or mucosal infections, caused by either bacteria or fungi $[6,26]$.

Antifungal PDT is an area of increasing interest, as research is advancing in answering fundamental questions regarding the photochemical and photophysical mechanisms involved in photoinactivation; producing new, potent and clinically compatible PS; and in understanding the effect of key microbial phenotypic multidrug resistance, virulence and pathogenesis determinants in photoinactivation. The novel concept of developing the non-vertebrate infection model in G. mellonella to explore the efficacy of antifungal PDT provides many competitive advantages [6]. 

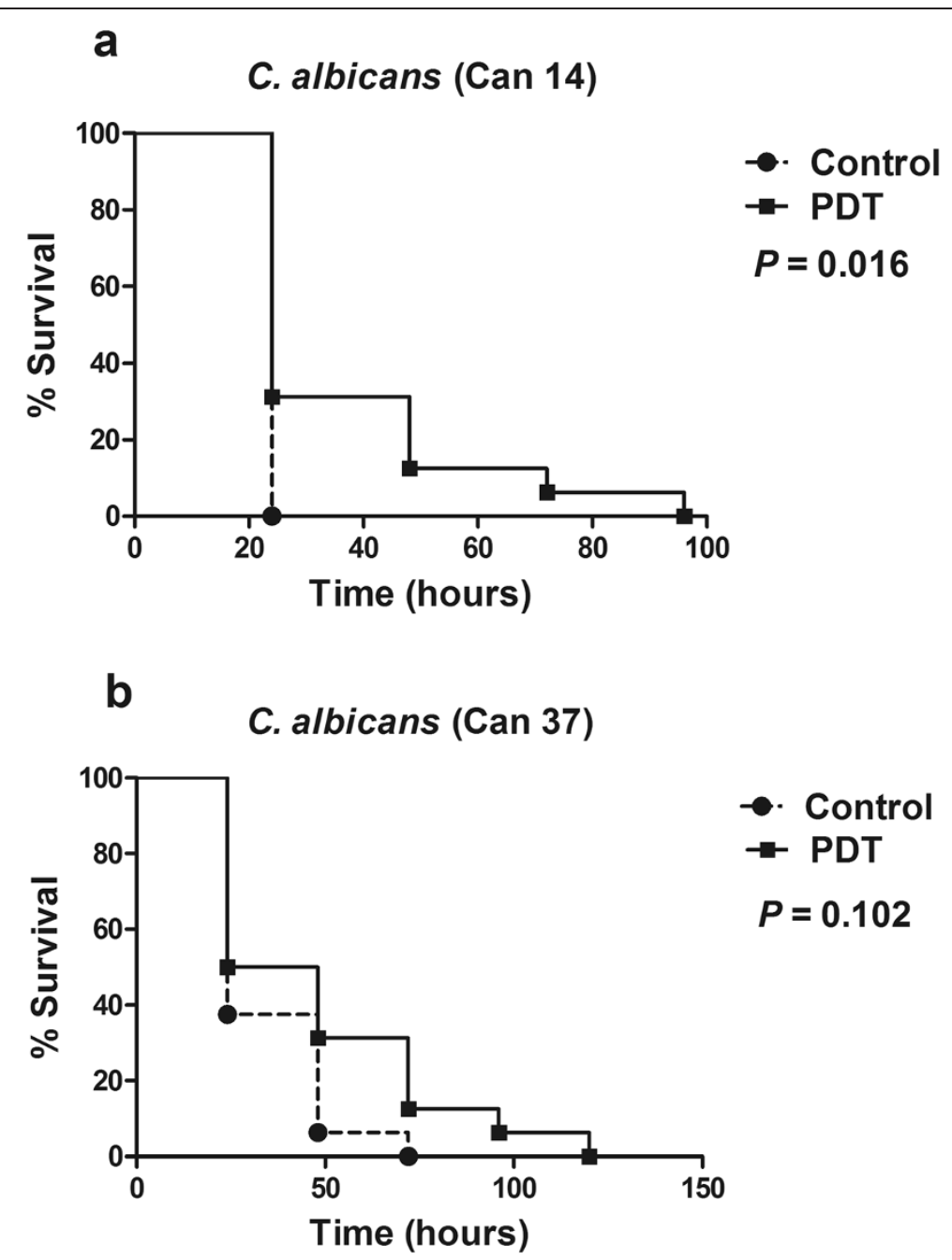

Figure 2 Killing of G. mellonella by C. albicans exposed to antimicrobial PDT. In the aPDT group, the larvae received the PS injection 90 min after the infection with $C$. albicans. In order to allow a good dispersion of the PS into the insect body, we waited at least 30 additional min after the PS injection prior to the light irradiation. A control group received PS without light exposure. Larvae were maintained at $37^{\circ} \mathrm{C}$. a) $\mathrm{C}$. albicans Can14 wild-type strain SC5314, b) C. albicans Can37 clinical isolate from oropharyngeal candidiasis and fluconazole resistant.

The use of the invertebrate model host has significant benefits when compared to mammalian animals: there are no ethical or legal concerns, no need for specialized feeding or housing facilities, the management of the animal is very easy and no anesthesia is needed, animals are inexpensive, and the use of large sample numbers in the same group are possible [27-30]. G. mellonella has been used to study host-pathogen interactions as an alternative host model to small mammals such as mice and rats [9,27-29,31-40].

Our laboratory pioneered the use of G. mellonella as a suitable invertebrate model host to study aPDT against Enterococcus faecium [19]. In the present study this approach to investigating aPDT was successfully expanded to include fungal pathogens. The optimal dose-response to $\mathrm{MB}$ mediated-PDT was evaluated and $0.9 \mathrm{~J} / \mathrm{cm}^{2}$ showed the best survival of G. mellonella caterpillars, as was found in the E. faecium study. The same limited non-toxic dosage of aPDT to G. mellonella was applied to treat larvae infected by strains of Candida albicans.

During the G. mellonella killing assays, groups infected by C. albicans that received aPDT treatment demonstrated prolonged survival when compared to groups that did not received treatment. However a statistically significant difference between PDT and control groups was observed only for C. albicans Can14 wild-type strain. When the infection was induced by a fluconazole resistant strain (Can37), a statistically significant difference between these groups was not observed. Despite the fact that PDT has been described as a potent agent against both antimicrobial-resistant and sensitive microorganisms [6] we observed that a fluconazoleresistant $C$. albicans strain was less sensitive to aPDT.

This difference has also been described in an in vitro study performed by Dovigo et al. [41]. These authors 


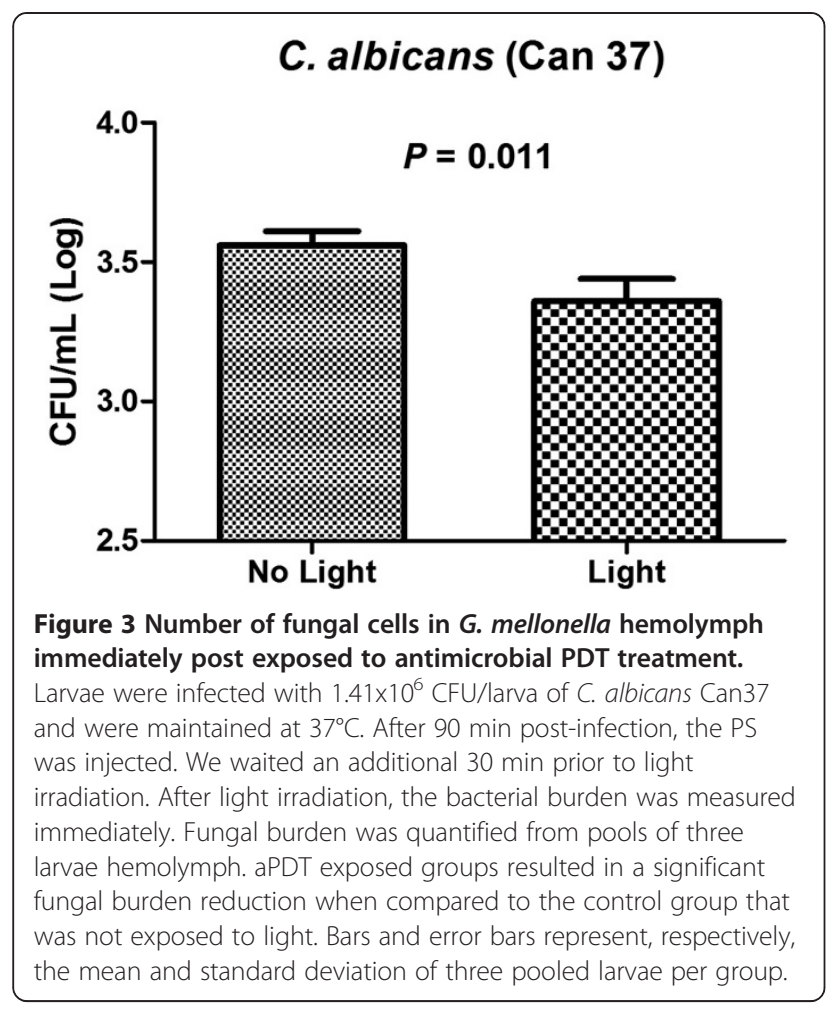

observed that fluconazole-resistant strains of C. albicans and C. glabrata showed reduced sensitivity to aPDT in comparison with reference strains susceptible to fluconazole, suggesting that resistance mechanisms of microorganisms to traditional antifungal drugs could reduce PDT effectiveness. According to Prates et al. [23], the resistance of Candida strains to fluconazole usually involves overexpression of cell membrane multidrug efflux systems belonging to the ATP-binding cassette (ABC) or the major facilitator superfamily (MFS) classes of transporters. The authors showed that the overexpression of both systems reduced MB uptake by fungal cells, as well as the killing effect of aPDT, suggesting that ABCs and MFSs are involved in the efficiency of aPDT mediated by MB and red light. In addition, Arana et al. [42] demonstrated that subinhibitory concentrations of fluconazole induced oxidative stress and a transcriptional adaptative response that was able to generate protection of $C$. albicans against subsequent challenges with oxidants. The mechanisms of protection against oxidative stress of fluconazole resistant $C$. albicans strain may have enhanced the resistance of $C$. albicans to oxidative damage caused by PDT.

In this study, we also evaluated the effects of aPDT on fungal cells in the hemolymph of G. mellonella larvae infected by fluconazole resistant C. albicans (Can37). Although this $C$. albicans strain had not shown a significant increase in survival rate in G. mellonella, it was observed that aPDT caused a reduction of the number of fungal cells in the hemolymph $(0.2 \mathrm{Log})$ with a statistically significant difference between aPDT and control groups. In addition, these data demonstrated that aPDT was able to reduce fungal cell viability immediately upon light exposure, suggesting that $C$. albicans cells were sensitive to aPDT, by the lethal oxidative damage of the singlet oxygen pathway, in the experimental candidiasis in the G. mellonella model. At the moment, all the aPDT studies performed in vivo were developed in vertebrate models of rats and mice using fluences of light much higher than the dose used in our work [43-45]. Using an oral candidiasis mice model, Costa and colleagues [44] found a reduction of $0.73 \mathrm{Log}$ in the fungal cells recovered after erythrosineand LED-mediated aPDT when a fluence of $14 \mathrm{~J} / \mathrm{cm}^{2}$ was applied. Dai et al. [45] also demonstrated that aPDT, with the combination of methylene blue and red light $(78 \mathrm{~J} /$ $\mathrm{cm}^{2}$ ), reduced (0.77 Log of CFU) the fungal burden in skin abrasion wounds in mice infected with C. albicans.

Patients with fungal infections are often treated with azole antifungal drugs, however Candida resistance to azoles has been detected in recent years. Several mechanisms of resistance have been reported including the overexpression of cell membrane multidrug efflux pumps previously cited, an alteration in the chemical structure of the demethylase enzyme, and the incorporation of alternative sterols to ergosterol within the cell membrane [23,24]. Giroldo et al. [25] suggested that MB-mediated aPDT caused damage to the cell membrane of the C. albicans cells. If the hypothesis that aPDT could affect the cell membrane is valid, the sequential use of aPDT with fluconazole could have a dual action on treating the infection. Conventional antimicrobial therapy could have aPDT as an adjunct or as an alternative [15]. The combination of PDT with antimicrobials has been used with success when compared to either isolated approach $[19,26,46]$. Kato et al. [43] verified that after exposure to sublethal aPDT, the minimal inhibitory concentration (MIC) of fluconazole against $C$. albicans was reduced compared to non-aPDT treated strains.

Of note, we observed that the G. mellonella larvae survival after infection by the fluconazole resistant $C$. albicans strain, was prolonged when fluconazole was administered before or after aPDT, in comparison to the use of fluconazole or PDT alone. We believe that due to the permeabilization of the fungal cell membrane by the sublethal PDT dose, fungal cells become more susceptible to fluconazole action. In addition, it has been suggested that the use of azoles can increase the oxidative stress promoted by PDT by contributing to ROS formation themselves [26]. Arana et al. [42] demonstrated that fluconazole was able to induce oxidative stress in C. albicans in a dose- and timedependent manner, suggesting that ROS play a role in the mechanism of action of azoles. The exact mechanism involved in increasing the survival of larvae infected by the fluconazole resistant $C$. albicans strain and exposed to 


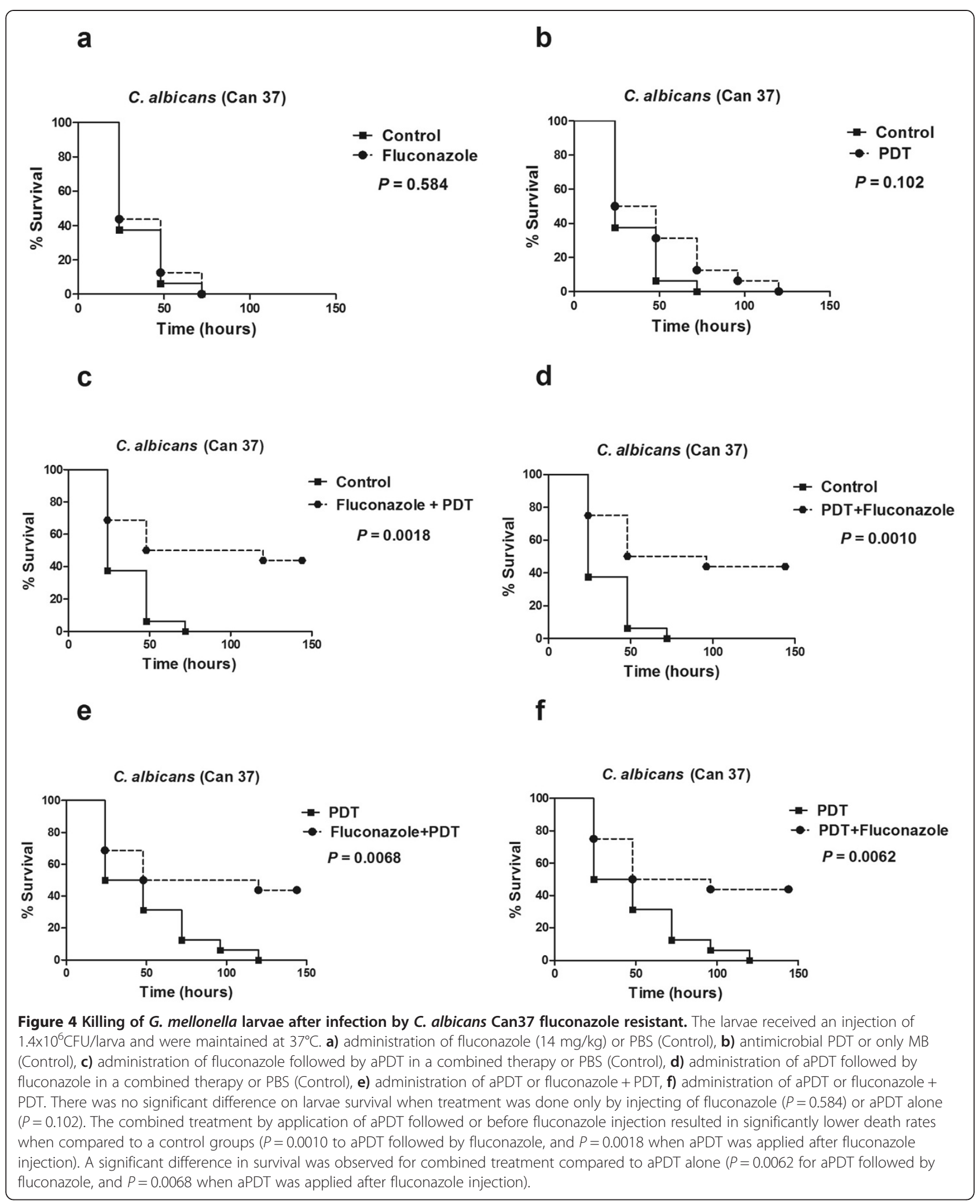


combined therapy of PDT and fluconazole remains to be clarified. Thus, comprehensive experiments are needed to better understand whether this process could be useful to treat antimicrobial resistant fungal infections.

In summary, the results obtained in this study showed that G. mellonella is a suitable model host to study the antifungal PDT in vivo. It is known that the G. mellonella model is not restricted to studies that examine aspects of the pathogenesis of fungal infections or antimicrobial therapies, but also can be used to the study of host defenses against fungal pathogens [30]. The insect immune response demonstrates a number of strong structural and functional similarities to the innate immune response of mammals and, in particular, insect haemocytes and mammalian neutrophils have been shown to phagocytose and kill pathogens in a similar manner [47]. Recent studies demonstrated that PDT can stimulate host defense mechanisms. Tanaka et al. [21] used a murine methicilin-resistant Staphylococcus aureus (MRSA) arthritis model and verified that the MB-mediated PDT exerted a therapeutic effect against a bacterial infection via the attraction and accumulation of neutrophils into the infected region. Neutrophils are among the first cells recruited to the illuminated area and their main function is to release enzymes for killing infectious organisms and secrete cytokines and other chemicals that promote inflammation [48]. In this study, the effects of aPDT on the immune system of G. mellonella were not investigated. Therefore, future studies need to be developed to understanding the action of aPDT and methylene blue in the haemocyte density and in the expression of a variety of antimicrobial peptides involved in immune responses of $G$. mellonella.

The key conclusion is that the G. mellonela - C. albicans system is a suitable model to study antifungal PDT and to explore combinatorial aPDT-based treatments. Thus, this invertebrate animal model host provides a novel approach to assess the effects of in vivo PDT, alone or in combination with antifungal compounds, on fungal infections without the difficulties of mammalian models.

\section{Authors' contributions}

Conceived and designed the experiments: JCJr, CPS, XT, BBF, MRH, EM. Performed the experiments: JCJr, CPS, XT, YW. Analyzed the data: JCJr, JCJ, AOCJ, GPT, MRH, EM. Contributed reagents/materials/analysis tools: MRH, EM. Wrote the paper: JCJr, JCJ, MRH, GPT, EM. All authors read and approved the final manuscript.

\footnotetext{
Acknowledgments

José Chibebe Junior thanks CAPES (PDEE 2507-11-0) for the scholarship during the PhD Program at Harvard Medical School. Xiaojiang Tan was supported by Science and Technology Planning Project of Guangdong Province, P.R. China (2011B080701091). Juliana C Junqueira thanks São Paulo Council of Research - FAPESP, Brazil (grant 12/19915-6). Research conducted in the Mylonakis Laboratory was supported by NIH (RO1 Al050875 to EM). Research conducted in the Hamblin Laboratory was supported by NIH (RO1 Al050875 to MRH) and US Air Force MFEL Program (FA9550-04-1-0079). George P Tegos was supported by the NIH (grant 5U54MH084690-02).
}

\section{Author details}

'Department of Biosciences and Oral Diagnosis, Univ Estadual Paulista/ UNESP, São José dos Campos, SP 12245000, Brazil. 'Division of Infectious Diseases, Massachusetts General Hospital, Boston, MA 02114, USA.

${ }^{3}$ Department of Restorative Dentistry, Faculty of Pindamonhangaba, Pindamonhangaba, SP 12422970, Brazil. "Wellman Center for Photomedicine, Massachusetts General Hospital, Boston, MA 02114, USA. ${ }^{5}$ Center for Lasers and Applications, Nuclear and Energy Research Institute, São Paulo, SP 05508000, Brazil. ${ }^{6}$ Huiqiao Department, Nanfang Hospital, Southern Medical University, Guangzhou 510515, People's Republic of China. ' 5 chool of Pharmacy, Second Military Medical University, Shanghai 200433, China. ${ }^{8}$ Department of Pathology and Center for Molecular Discovery, University of New Mexico, Albuquerque, NM 87131, USA. 'Department of Dermatology, Harvard Medical School, Boston, MA 02114, USA. ${ }^{10}$ Harvard-MIT Division of Health Sciences and Technology, Cambridge, MA 02139, USA. " Warren Alpert Medical School, Brown University/Rhode Island and Miriam Hospitals, Providence, RI 02903, USA.

Received: 18 April 2013 Accepted: 17 September 2013 Published: 1 October 2013

\section{References}

1. Chabrier-Rosello Y, Giesselman BR, De Jesus-Andino FJ, Foster TH, Mitra S, Haidaris CG: Inhibition of electron transport chain assembly and function promotes photodynamic killing of Candida. J Photochem Photobiol B 2010, 99:117-125.

2. Thein ZM, Seneviratne CI, Samaranayake YH, Samaranayake LP: Community lifestyle of Candida in mixed biofilms: a mini review. Mycoses 2009, 52:467-475.

3. Junqueira JC, Fuchs BB, Muhammed M, Coleman JJ, Suleiman JM, Vilela SF, Costa AC, Rasteiro VM, Jorge AO, Mylonakis E: Oral Candida albicans isolates from HIV-positive individuals have similar in vitro biofilmforming ability and pathogenicity as invasive Candida isolates. BMC Microbiol 2011, 11:247.

4. Cowen LE, Singh SD, Kohler JR, Collins C, Zaas AK, Schell WA, Aziz H, Mylonakis E, Perfect JR, Whitesell L, et al: Harnessing Hsp90 function as a powerful, broadly effective therapeutic strategy for fungal infectious disease. Proc Natl Acad Sci USA 2009, 106:2818-2823.

5. Douglas LJ: Candida biofilms and their role in infection. Trends Microbiol 2003, 11:30-36.

6. Dai T, Fuchs BB, Coleman JJ, Prates RA, Astrakas C, St Denis TG, Ribeiro MS, Mylonakis E, Hamblin MR, Tegos GP: Concepts and principles of photodynamic therapy as an alternative antifungal discovery platform. Front Microbiol 2012, 3:120.

7. Niimi M, Firth NA, Cannon RD: Antifungal drug resistance of oral fungi. Odontology 2010, 98:15-25.

8. Coleman JJ, Okoli I, Tegos GP, Holson EB, Wagner FF, Hamblin MR, Mylonakis E: Characterization of plant-derived saponin natural products against Candida albicans. ACS Chem Biol 2010, 5:321-332.

9. Fuchs BB, Eby J, Nobile CJ, El Khoury JB, Mitchell AP, Mylonakis E: Role of filamentation in Galleria mellonella killing by Candida albicans. Microbes Infect 2010, 12:488-496.

10. Zhang X, De Micheli M, Coleman ST, Sanglard D, Moye-Rowley WS: Analysis of the oxidative stress regulation of the Candida albicans transcription factor, Cap1p. Mol Microbiol 2000, 36:618-629.

11. Williams DW, Kuriyama T, Silva S, Malic S, Lewis MA: Candida biofilms and oral candidosis: treatment and prevention. Periodontol 2000 2011, 55:250-265.

12. Kusch H, Engelmann S, Albrecht D, Morschhauser J, Hecker M: Proteomic analysis of the oxidative stress response in Candida albicans. Proteomics 2007, 7:686-697.

13. Wang Y, Cao YY, Jia XM, Cao YB, Gao PH, Fu XP, Ying K, Chen WS, Jiang YY: Cap1p is involved in multiple pathways of oxidative stress response in Candida albicans. Free Radic Biol Med 2006, 40:1201-1209.

14. Alonso-Monge R, Navarro-Garcia F, Roman E, Negredo Al, Eisman B, Nombela C, Pla J: The Hog1 mitogen-activated protein kinase is essential in the oxidative stress response and chlamydospore formation in Candida albicans. Eukaryot Cell 2003, 2:351-361.

15. Chabrier-Rosello Y, Foster TH, Mitra S, Haidaris CG: Respiratory deficiency enhances the sensitivity of the pathogenic fungus Candida to photodynamic treatment. Photochem Photobiol 2008, 84:1141-1148. 
16. Fuchs BB, Tegos GP, Hamblin MR, Mylonakis E: Susceptibility of Cryptococcus neoformans to photodynamic inactivation is associated with cell wall integrity. Antimicrob Agents Chemother 2007, 51:2929-2936.

17. Denis TGS, Dai T, Izikson L, Astrakas C, Anderson RR, Hamblin MR, Tegos GP: Antimicrobial photoinactivation as an evolving and emerging discovery strategy against infectious disease. Virulence 2011, 2:509-520.

18. Desalermos A, Fuchs BB, Mylonakis E: Selecting an invertebrate model host for the study of fungal pathogenesis. PLoS Pathog 2012, 8:e1002451.

19. Chibebe Junior J, Fuchs BB, Sabino CP, Junqueira JC, Jorge AOC, Ribeiro MS, Gilmore MS, Rice LB, Tegos GP, Hamblin MR, Mylonakis E: Photodynamic and antibiotic therapy impair the pathogenesis of Enterococcus faecium in a whole animal insect model. Plos One 2013, 8:e55926.

20. Gillum AM, Tsay EY, Kirsch DR: Isolation of the Candida albicans gene for orotidine-5-phosphate decarboxylase by complementation of $S$. cerevisiae Ura3 and E. coli PyrF mutations. Mol Gen Genet 1984, 198:179-182.

21. Tanaka M, Mroz P, Daí T, Huang L, Morimoto Y, Kinoshita M, Yoshirara Y, Nemoto K, Shinomiya N, Seki S, Hamblin MR: Photodynamic therapy can induce a protective innate immune response against murine bacterial arthritis via neutrophil accumulation. Plos One 2012, 7:e39823.

22. Dougherty TJ, Gomer CJ, Henderson BW, Jori G, Kessel D, Korbelik M, Moan J, Peng Q: Photodynamic therapy. J Natl Cancer Inst 1998, 90:889-905.

23. Prates RA, Kato IT, Ribeiro MS, Tegos GT, Hamblin MR: Influence of multidrug efflux systems on methylene blue-mediated photodynamic inactivation of Candida albicans. J Antimicrob Chemother 2011, 66:1525-1532.

24. Rautemaa R, Ramage G: Oral candidosis-clinical challenges of a biofilm disease. Crit Rev Microbiol 2011, 37(4):328-336.

25. Giroldo LM, Felipe MP, Oliveira MA, Munin E, Alves LP, Costa MS Photodynamic antimicrobial chemotherapy (PACT) with methylene blue increases membrane permeability in Candida albicans. Lasers Med Sci 2009, 24:109-112.

26. Snell SB, Foster TH, Haidaris CG: Miconazole induces fungistasis and increases killing of Candida albicans subjected to photodynamic therapy (dagger). Photochem Photobiol 2011, 88:596-603.

27. Aperis G, Fuchs BB, Anderson CA, Warner JE, Calderwood SB, Mylonakis E: Galleria mellonella as a model host to study infection by the Francisella tularensis live vaccine strain. Microbes Infect 2007, 9:729-734.

28. Olsen RJ, Watkins ME, Cantu CC, Beres SB, Musser JM: Virulence of serotype M3 Group A Streptococcus strains in wax worms (Galleria mellonella larvae). Virulence 2011, 2:111-119.

29. Peleg AY, Jara S, Monga D, Eliopoulos GM, Moellering RC Jr, Mylonakis E: Galleria mellonella as a model system to study Acinetobacter baumannii pathogenesis and therapeutics. Antimicrob Agents Chemother 2009, 53:2605-2609.

30. Fuchs $B B$, Mylonakis $E$ : Using non-mammalian hosts to study fungal virulence and host defense. Curr Opin Microbiol 2006, 9:346-351.

31. Abranches J, Miller JH, Martinez AR, Simpson-Haidaris PJ, Burne RA, Lemos $J A$ : The collagen-binding protein $\mathrm{Cnm}$ is required for Streptococcus mutans adherence to and intracellular invasion of human coronary artery endothelial cells. Infect Immun 2011, 79:2277-2284.

32. Champion OL, Cooper IA, James SL, Ford D, Karlyshev A, Wren BW, Duffield M, Oyston PC, Titball RW: Galleria mellonella as an alternative infection model for Yersinia pseudotuberculosis. Microbiology 2009, 155:1516-1522.

33. Desbois AP, Coote PJ: Wax moth larva (Galleria mellonella): an in vivo model for assessing the efficacy of antistaphylococcal agents. J Antimicrob Chemother 2011, 66:1785-1790.

34. Gaddy JA, Arivett BA, McConnell MJ, Lopez-Rojas R, Pachon J, Actis LA: Role of Acinetobactin-mediated iron acquisition functions in the interaction of Acinetobacter baumannii strain ATCC 19606T with human lung epithelial cells, Galleria mellonella caterpillars, and mice. Infect Immun 2012, 80:1015-1024.

35. Jander $G$, Rahme $L G$, Ausubel FM: Positive correlation between virulence of Pseudomonas aeruginosa mutants in mice and insects. J Bacteriol 2000, 182:3843-3845.

36. Lebreton F, Le Bras F, Reffuveille F, Ladjouzi R, Giard JC, Leclerca R, Cattoir V: Galleria mellonella as a model for studying Enterococcus faecium host persistence. J Mol Microbiol Biotechnol 2011, 21:191-196.

37. Miyata S, Casey M, Frank DW, Ausubel FM, Drenkard E: Use of the Galleria mellonella caterpillar as a model host to study the role of the type III secretion system in Pseudomonas aeruginosa pathogenesis. Infect Immun 2003, 71:2404-2413.

38. Mylonakis E, Moreno R, El Khoury JB, Idnurm A, Heitman J, Calderwood SB, Ausubel FM, Diener A: Galleria mellonella as a model system to study Cryptococcus neoformans pathogenesis. Infect Immun 2005, 73:3842-3850.
39. Yasmin A, Kenny JG, Shankar J, Darby AC, Hall N, Edwards C, Horsburgh MJ: Comparative genomics and transduction potential of Enterococcus faecalis temperate bacteriophages. J Bacteriol 2010, 192:1122-1130.

40. Michaux C, Sanguinetti M, Reffuveille F, Auffray Y, Posteraro B, Gilmore MS, Hartke A, Giard JC: SlyA is a transcriptional regulator involved in the virulence of Enterococcus faecalis. Infect Immun 2011, 79:2638-2645.

41. Dovigo LN, Pavarina AC, Mima EG, Giampaolo ET, Vergani CE, Bagnato VS: Fungicidal effect of photodynamic therapy against fluconazole-resistant Candida albicans and Candida glabrata. Mycoses 2011, 54:123-130.

42. Arana DM, Nombel C, Pla J: Fluconazole at subinhibitory concentrations induces the oxidative- and nitrosative-response genes TRR1, GRE2 and YHB1, and enhances the resistance of Candida albicans to phagocytes. J Antimicrob Chemother 2010, 65:54-62.

43. Kato IT, Prates RA, Sabino CP, Fuchs BB, Tegos GP, Mylonakis E, Hamblin MR, Ribeiro MS: Antimicrobial photodynamic inactivation inhibits Candida albicans virulence factors and reduces in vivo pathogenicity. Antimicrob Agents Chemother 2012, 57:445-451.

44. Costa AC, Campos-Rasteiro VM, Da Silva Hashimoto ES, Araujo CF, Pereira CA, Junqueira JC, Jorge AO: Effect of erythrosine- and LED-mediated photodynamic therapy on buccal candidiasis infection of immunosuppressed mice and Candida albicans adherence to buccal epithelial cells. Oral Surg Oral Med Oral Pathol Oral Radiol 2012, 114:67-74

45. Dai T, Arce VJB, Tegos GP, Hamblin MR: Blue dye and red light, a dynamic combination for prophylaxis and treatment of cutaneous Candida albicans infections in mice. Antimicrob Agents Chemother 2011, 55:5710-5717.

46. Di Poto A, Sbarra MS, Provenza G, Visai L, Speziale P: The effect of photodynamic treatment combined with antibiotic action or host defence mechanisms on Staphylococcus aureus biofilms. Biomaterials 2009, 30:3158-3166.

47. Fallon JP, Troy N, Kavanagh K: Pre-exposure of Galleria mellonella larvae to different doses of Aspergillus fumigatus conidia causes differential activation of cellular and humoral immune responses. Virulence 2011, 2:413-421.

48. Huang YY, Tanaka M, Vecchio D, Garcia-Diaz M, Chang J, Morimoto Y, Hamblin MR: Photodynamic therapy induces an immune response against a bacterial pathogen. Expert Rev Clin Immunol 2012, 8:479-494.

doi:10.1186/1471-2180-13-217

Cite this article as: Chibebe Junior et al:: Selective photoinactivation of Candida albicans in the non-vertebrate host infection model Galleria mellonella. BMC Microbiology 2013 13:217.

\section{Submit your next manuscript to BioMed Central and take full advantage of:}

- Convenient online submission

- Thorough peer review

- No space constraints or color figure charges

- Immediate publication on acceptance

- Inclusion in PubMed, CAS, Scopus and Google Scholar

- Research which is freely available for redistribution

Submit your manuscript at www.biomedcentral.com/submit
C Biomed Central 